\title{
||||||||||||||||||||||||||||||||||||||||||||||||||||||||||||||||||.
}

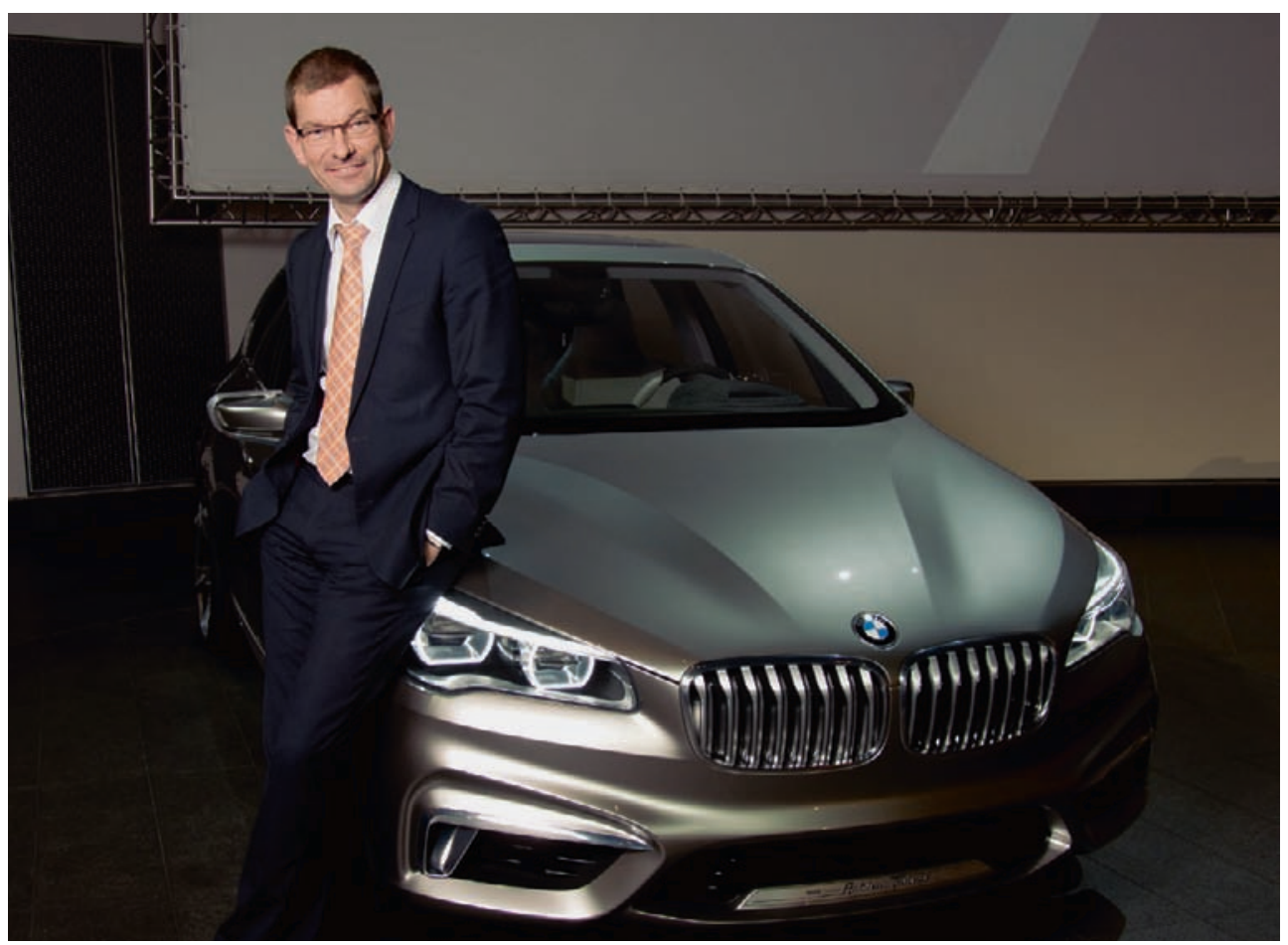

MARKUS DUESMANN

Leiter Antriebsentwicklung BMW AG

\section{RIGHTSIZING IST MEHR ALS DOWNSIZING}

Die Erfolge der Automobilindustrie in der Reduktion der $\mathrm{CO}_{2}^{-}$ Emissionen ihrer Fahrzeugflotten beruht maßgeblich auf dem Downsizing von Verbrennungsmotoren. Nachdem erkannt wurde, dass bis weit in die nächsten Jahrzehnte der Verbrennungsmotor als Basis für den Fahrzeugantrieb erhalten bleiben wird, stellt sich die Frage, wie zukunfts- und ausbaufähig der Downsizing-Ansatz ist.

In den letzten Jahren wurden zahlreiche Saugmotoren sukzessive durch aufgeladene und im Hubraum verkleinerte Aggregate ersetzt. Dabei konnte in den meisten Fällen dem Kunden ein überzeugendes Angebot gemacht werden: mehr Fahrleistung bei weniger Verbrauch. In Summe ist das eine Erfolgsstory, die zeigt, dass der Verbrennungsmotor trotz über 100-jähriger Geschichte seine Anpassungsfähigkeit nicht verloren hat.

Das Mittel „Downsizing“ hat allerdings auch einige Nebenwirkungen: Der Unterschied zwischen Zyklusverbrauch und Realverbrauch kann je nach Fahrprofil höher sein als bei den abgelösten Vorgängerkonzepten mit größeren Hubräumen. Wird Downsizing mit einer Reduktion der Zylinderzahl kombiniert, müssen Antrieb und Gesamtfahrzeug befähigt werden, mit der höheren Drehungleichförmigkeit des Motors umzugehen.

Die eigentliche Herausforderung für das Downsizing-Programm steht allerdings noch bevor: die anstehenden Veränderungen und Verschärfungen der weltweiten Verbrauchs- und Emissionsgesetzgebungen. Die neuen Verbrauchszyklen, konkret
WLTP, bedeuten einen Betrieb der Motoren bei durchschnittlich höheren Lasten und damit im Ergebnis höheren Verbräuchen. Auch in der Emissionierung können zu kleine Brennräume zu erhöhten Rohemissionen führen, die den Verschärfungen der Euro-6- und RDE-Gesetzgebung nicht mehr genügen. Es gilt ein Optimum beim Downsizing für die jeweilige Produktanwendung zu finden, also ein „Rightsizing“ des Motors. Verbrauch, Emissionen, Kosten und nicht zuletzt Funktions- und Fahreigenschaften sind dabei erneut in Einklang zu bringen.

Diesem Gedanken folgend hat sich die BMW Group entschlossen, eine neue Otto-/Diesel-Motorenfamilie aufzusetzen, die in knapp einem Jahr in Serie gehen wird. Der Dreizylinder mit 1,5 1 Hubraum spiegelt am deutlichsten den RightsizingGedanken wider. Er wird allein oder in Kombination mit Elektroantrieben in sehr verschiedenen Produkten zum Einsatz kommen. Ein Beispiel ist der kürzlich vorgestellte BMW Concept Active Tourer, mit dem BMW nicht nur in ein neues Produktsegment vorstößt, sondern auch das Beste aus zwei Welten in seinem Antrieb vereint: einen aufgeladenen Dreizylinder-1,5-1Ottomotor mit Valvetronic-Technologie und eine Hybrid-Synchron-Elektromaschine aus hauseigener Entwicklung. Beides zusammen sorgt für überragende Fahrleistungen bei minimalem $\mathrm{CO}_{2}$-Ausstoß von unter $60 \mathrm{~g} / \mathrm{km}$.

Rightsizing zeigt für die Verbrennungsmotoren den Weg in die Zukunft auf. Es lohnt sich, intensiv daran zu arbeiten. 